\title{
Study on the Construction of Featured Education Resource Database in the National Universities of China
}

\author{
Jun Ma \\ Northwest University for Nationalities \\ NWUN \\ Lanzhou, China \\ 364221500@qq.com
}

\begin{abstract}
The article studied the construction of educational database with Chinese ethnic characteristic for the ethnic universities. It addressed the basic framework, principles, procedures and contents of the construction, and put forward the standards for the construction and specific tasks and requirements for the constructors in order to realize the digital heritage and sharing of the ethnic cultural resources in China.
\end{abstract}

Keywords-ethnic university; characteristic database; data resource; CALIS

\section{INTRODUCTION}

China is a multinational country and includes 55 ethnic minorities. Each nationality is equipped with its unique historical culture and inhabitation and cultivates various talents for minority areas. China has established different ethnic schools at different levels since 1949, covering basic education, higher education and vocational education and involving 17 ethnic universities[1]. In order to better serve national educational business and inherit minority culture, these ethnic universities have constantly developed and established data resource library with national cultural characteristics based on school libraries to better serve teachers and students in an information means[2]. These characteristic resource libraries take certain shape at present, as shown in Table I:

TABLE I.

CHARACTERISTIC DATABASE OF ETHNIC SCHOOLS

\begin{tabular}{|c|c|c|}
\hline Ethnic School & $\begin{array}{c}\text { Quantity of Characteristic } \\
\text { Database }\end{array}$ & Name of Characteristic Database \\
\hline Inner Mongolia University & 4 & $\begin{array}{l}\text { Cultural and art resource library of the Mongol nationality } \\
\text { Tourism resource library of Inner Mongolia style } \\
\text { Culture collection database of ancient time } \\
\text { Resource library of cultural relic exposition of Inner Mongolia }\end{array}$ \\
\hline $\begin{array}{l}\text { Northwest University for } \\
\text { Nationalities }\end{array}$ & 3 & $\begin{array}{c}\text { Special ethnic study database in Gansu } \\
\text { Bibliographical database of ethnic research literature } \\
\text { Bibliography database of ancient books in library }\end{array}$ \\
\hline $\begin{array}{l}\text { Southwest University for } \\
\text { Nationalities }\end{array}$ & 4 & $\begin{array}{c}\text { Qiang bibliographic database } \\
\text { Western Xia database } \\
\text { Yi literature database } \\
\text { Research literature database of ethnic history }\end{array}$ \\
\hline Xinjiang University & 6 & $\begin{array}{c}\text { Chagatai title document resource library } \\
\text { Full-text database of characteristic journals in northwest minority research } \\
\text { center } \\
\text { Achievement database of northwest minority research center in Xinjiang } \\
\text { University } \\
\text { Xinjiang research database } \\
\text { Local bibliography resource library of Sinkiang } \\
\text { Local bibliography resource library index journal library }\end{array}$ \\
\hline Tibet University & 3 & $\begin{array}{c}\text { Bibliography number of Tibetology } \\
\text { Special full-text database of Tibetology } \\
\text { Full-text database of Tibetology books in library }\end{array}$ \\
\hline
\end{tabular}

In the paper, thought, framework, process and content of the construction of characteristic database in the paper were described.

Philosophy and Social Science Planning Project of Gansu Province - Research on the Development and Construction of Featured Education Resources in Gansu Minorities Areas (Grant NO. YB017) .

\section{CONSTRUCTION THOUGHTS}

First, Characteristic database construction of ethnic schools refers to special characteristic database and planning scheme of literature guarantee system in other regions to establish characteristic guarantee system of ethnic schools. It 
should integrally plan resource construction of characteristic database and organize and encourage colleges and universities with advantage of discipline and characteristics of document resources to participate.

Second, Characteristic resource sharing platform should be established preliminarily and service work of characteristics of ethnic schools should be standardized. Taken retrieval of characteristic database and application service as core, central website of characteristic database construction program of ethnic schools is established.

Third, Standard and normative system of characteristic database of ethnic schools should be established and study on safety problems should be strengthened.

Fourth, it should conduct study on co-construction and sharing model of characteristic database of ethnic schools to promote co-construction and sharing of characteristic database of ethnic schools.

\section{DATABASE FRAMEWORK}

Overall structure of characteristic database construction of ethnic schools is "project center system of characteristic database construction of ethnic schools" - "participation in local characteristic database system" to form "one-network and multi-library" layout[3]. "One network" refers to distributed information service network combined with central network and services sites of characteristic library of various ethnic schools.

Central website connection and sharing characteristic literature and information resources of ethnic schools provide networked, digitalized, individualized and "one-stop" literature and information service for users[4]. Sharing database established in center integrates and combines metadata information and object database of characteristic resources built by various schools. Its structure is as shown in Fig.1.

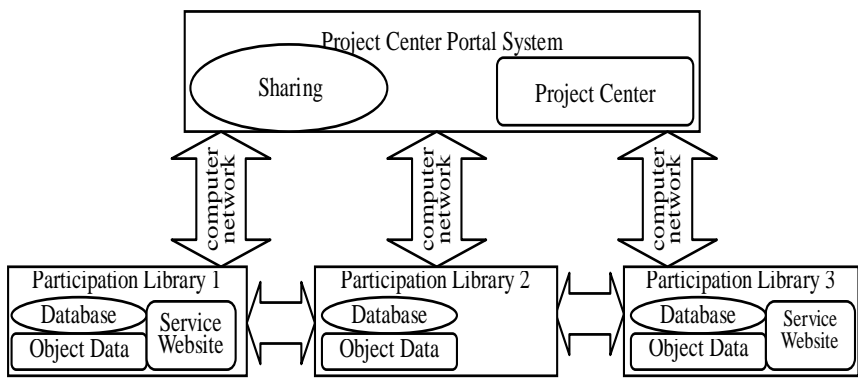

Fig. 1.Frame Diagram of Characteristic Database of Ethnic Schools

\section{CONSTRUCTION PRINCIPLE}

\section{A. Demand Principle}

Construction selection of characteristic database should be based on demand of learner and should face teaching and scientific research and meet actual requirement of local economic construction service and practical value and requirement should be taken into account.

\section{1) Reader's Demand}

Final target of database construction should provide larger convenience for more readers. So database construction is meaningless without reader's demand.

\section{2) Scientific Research Demand}

It should highlight key disciplines and professional characteristics of schools and should be closely related to requirement of teaching and scientific research. Moreover, it should consider whether it can promote the teaching and scientific research and whether it can create benefits for social development and economic construction.

\section{B. Quality Principle}

Quality of characteristic database is the representation of vitality of overall database and is the key to provide adequate guarantee for resource sharing of database. High-quality characteristic database should be comprehensive, professional and specialized [5]. Comprehension refers to information collected from certain field or topic; profession refers to pay attention to correlation of literature but should not include unnecessary data for pursing size of database; specialization means that processing of data should reflect essential features of literatures and should be specialized and accurate. Database with poor quality is only harmful for teaching and scientific research, so it is no need to establish.

\section{Principle of Convenience}

Characteristic database is built to furthest satisfy the demand of users for documental information. Database assessment is mainly related to whether the result is reasonable, feasible and practical. Therefore, before establishing basement, it should study the resource distribution status of basement in our country and the region to determine the method, content and size of the basement. Meanwhile, it should observe whether software and hardware that support working of database can support full-text research and multimedia service [6]. Finally, it is helpful for readers to conveniently, swiftly and accurately searching and applying literature resources.

\section{Principle of Standardization and Normalization}

It includes standard of data processing, collection, operation, review, transmission, exchange and maintenance. Data standardization and normalization is premise of guaranteeing data quality and forming data networking and resource sharing. Therefore, characteristic database construction must strictly follow relevant standard and format to build database to ensure that readers can accurately, completely and rapidly review required information resources and ensure that various libraries online can realize data compatibility. Using the Template

\section{E. Principle of Joint Database Construction}

Joint database construction is main approach to build characteristic database with high quality. Libraries of various ethnic schools should jointly set up library with libraries in different regions and different systems based on pushing network resource sharing and give play of power of group and 
complement each other's advantages of people and property [7].

\section{Construction Procedures}

\section{A. Database Selection}

Database selection of various participation libraries should meet the requirement for construction target and selection can take special resources of key disciplines, ethnic schools, ethnic regional economy, ethnic local culture, ethnic competitive industries and other special resources in library. In terms of selection, it should pay special attention to handle relationship between "characteristic" and "practice". As the participation library, it should consider the goal of library construction and database construction can provide assistance for discipline construction of school.

\section{B. Formation of Digitalized Standard}

Information resource construction standard of participation library applies major project of platform construction based on science and technology platform of ministry of science and technology: 16 standard specifications in the research result of Standard and Standardized Construction of Digital Library in our Country (http: cdls .nstl .gov .cn)[8]. This standard specification is also applied by CALIS agency. As digital resources of various participation libraries are abundant and above specification only provides description in broad sense. For example, participation library should make more accurate description for some concrete application and it can be expanded appropriately, but expansion lies in that semantics of original core metadata should not be destroyed and added element should not be similar to existing elements semantically.

Participation library can download description standard template of different resources from project center website. Description item required by template should be described. It can make choice on other recommended elements according to specific situations and should give introduction to relevant materials when making choice.

If above standard cannot adapt to practical application of the library and new standard is required to be established, it needs to provide relevant material to research group for recording.

\section{Selection of Local Database Construction System}

Database should be equipped with good software and hardware platform and library construction system should be possessed of fundamental functions of searching, issuing and system management. System management function involves user management and authentication, authority management, statistics, logging and system maintenance. Characteristic database center website proposes to apply OAI to collect metadata of participation library, so local library construction system needs to support OAI Data Provider at least.

\section{Selection of Resources}

Database should covers various literatures related to selection, including books, journal, patent, standard, conference papers, academic dissertation, rare ancient book, rubbing and map, photograph manuscript and video and audio recording. Actual library construction content should not be less than three literature types and one-time literature (such as full text, picture, audio and video materials) should not be less than $30 \%$.

For resource collection, it should pay attention to quality of material and should take two aspects into account, comprehension and reliability. Instead of the idea that more data will be better, comprehension means that it should judge whether data is complete or not in the range based on objective and use object of database construction. Reliability means that it needs to judge whether data in the database is wrong or not is of reference significance and whether it can reflect the last result and view.

\section{E. Resource Scale}

Database should be equipped with certain data size. During the acceptance, data size in the database should show a certain scale and can be put into use. Resource size should be considered when participating in establishing library. Meanwhile, it should strengthen the capacity of resource guarantee.

\section{F. Service Website Construction}

Service website of database should be an independent subweb under library website and should meet the requirement of frame system and column setting proposed by manager, showing unified page style. Processed data is issued through this website to provide data retrieval and other services for users. Other library of participation library can review metadata of this website and the center can visit this website.

\section{G. Device and Staff}

Applicant should be equipped with excellent devices and staffs and strong network communication ability for users to visit network. Project group should be participated in by professional and technician with subject background.

\section{H. Joint Construction}

It encourage many units to jointly establish characteristic thematic database with same subject and units participating in the project should comply with the rule of characteristic database resource sharing of ethnic schools of ethnic schools and the complete project should be regarded as collection construction result of characteristic database of ethnic schools. Regarding metadata, construction unit should also provide open and free retrieval in WEB to participation library of characteristic database of ethnic schools.

\section{Content of DAtabase Construction}

\section{A. Planning of Characteristic Database of Ethnic Schools}

Through construction of characteristic database resource, it forms database group with characteristics of national key discipline, dominant specialties, regional economy, local culture and competitive industries to serve national higher 
education and national and regional modernization construction.

\section{B. Construction of Resource Sharing Platform of Special Data}

Construction of characteristic data resource sharing platform takes portal web construction of project center and meta-database development as subject to realize sharing of characteristic data source and provide "one-stop" characteristic data resource service for users based on meta database of different schools and result of object database construction.

\section{Data Standardized Specification}

It should comply with standard and specification of China's digital library and CALIS characteristic database construction and combine with real condition of ethnic schools to build specification and standard of characteristic database of ethnic schools.

\section{Data Safety}

System should be designed comprehensively in the aspect of safety, including operating system, Web server and database and application delivery management system. It should guarantee system safety, digital resource access security and data security.

\section{E. Establishment of Resource Sharing Model for Characteristic Data}

Resource sharing rule of characteristic data of ethnic schools should be established. After the establishment of database, participation library, as common result of subject construction, should not only provide metadata to center but also should provide open and free retrieval of WEB to various participation libraries.

\section{F. Establishment of Sustainable Development Mechanism}

It includes management model, project operation model and system operation model. The project applies central management with relatively unified database construction standard. It should separately set up database for various participation libraries and special data of various schools should be managed by means of subproject and given subsidy.

\section{G. Establishment of Assessment on Subproject}

According to relevant standard and evaluation of CALIS on thematic database, real condition in characteristic database construction of ethnic colleges should be combined to establish a set of method for subproject assessment and to evaluate and accept subproject.

\section{CONCLUSION}

Characteristic database is the important support of ethnic schools for providing deep information consulting service and is development orientation of data library and effective method for digital inheritance of national culture. Various ethnic schools need to rely on characteristic database construction to train teams, expand thought and promote the deep development of information consulting work and provide high-quality information guarantee service for teaching and scientific research of schools.

\section{ACKNOWLEDGMENT}

This research was financially supported by the Philosophy and Social Science Planning Project of Gansu ProvinceResearch on the Development and Construction of Featured Education Resources in Gansu Minorities Areas (Grant NO. YB017)

\section{REFERENCES}

[1] Liu Li, Investigation and Analysis on Characteristic Database Construction of University Library in Ningxia , Library Journal, vol.11, pp. 37-41,2014 . In Chinese

[2] Zeng Ya, Study on Assessment Indicator System of Characteristic Database, Sichuan Library Journal, vol.6, pp. 55-60, 2014 . In Chinese

[3] Liu Kuibo, Analysis on Characteristic Database and Relevant Concept , Library Construction. vol.4, pp. 165-171,2015. In Chinese

[4] Lu. H, Construction and consideration of characteristic database system, Jiangsu science and technology information, vol.2, pp.17-21, 2009. In Chinese

[5] Wu Gao, Wei Nanhua. Study on Copyright Infringement Risk and Solution of Characteristic Database, Library, vol.2, pp. 73-80,2014 . In Chinese

[6] Zhou Huan, Zhang Yingcun. Exploration on Principle of Characteristic Database Construction of Library . Old Area Construction, vol.14, pp. 37-42, 2010 . In Chinese

[7] Li Juan, Reflection on Characteristic Database Construction of Library , Lantai World, vol.4, pp. 17-22, 2010 . In Chinese

[8] Tang. J, Discussion on some problems about the construction of characteristic database in University Library.Hebei Science and Technology Figure, vol.3, pp.120-124, 2012. In Chinese 